\title{
UM206, a selective Frizzled antagonist, attenuates adverse remodeling after myocardial infarction in swine
}

\author{
André Uitterdijk 1,4, Kevin CM Hermans ${ }^{2,4}$, Daphne PM de Wijs-Meijler ${ }^{1}$, Evangelos P Daskalopoulos ${ }^{2}$, Irwin K Reiss ${ }^{3}$, \\ Dirk J Duncker ${ }^{1}$, W Matthijs Blankesteijn² and Daphne Merkus ${ }^{1}$
}

Modulation of Wnt/Frizzled signaling with UM206 reduced infarct expansion and prevented heart failure development in mice, an effect that was accompanied by increased myofibroblast presence in the infarct, suggesting that Wnt/Frizzled signaling has a key role in cardiac remodeling following myocardial infarction (MI). This study investigated the effects of modulation of Wnt/Frizzled signaling with UM206 in a swine model of reperfused Ml. For this purpose, seven swine with MI were treated with continuous infusion of UM206 for 5 weeks. Six control swine were treated with vehicle. Another eight swine were sham-operated. Cardiac function was determined by echo in awake swine. Infarct mass was estimated at baseline by heart-specific fatty acid-binding protein ELISA and at follow-up using planimetry. Components of Wnt/Frizzled signaling, myofibroblast presence, and extracellular matrix were measured at follow-up with qPCR and/or histology. Results show that UM206 treatment resulted in a significant decrease in infarct mass compared with baseline $(-41 \pm 10 \%)$, whereas infarct mass remained stable in the Control-MI group $(+3 \pm 17 \%)$. Progressive dilation of the left ventricle occurred in the Control-MI group between 3 and 5 weeks after Ml, while adverse remodeling was halted in the UM206-treated group. mRNA expression for Frizzled-4 and the Frizzled co-receptor LRP5 was increased in UM206-treated swine as compared with Control-MI swine. Myofibroblast presence was significantly lower in infarcted tissue of the UM206-treated animals $(1.53 \pm 0.43 \%$ vs $3.38 \pm 0.61 \%)$ at 5 weeks follow-up. This study demonstrates that UM206 treatment attenuates adverse remodeling in a swine model of reperfused Ml, indicating that Wnt/Frizzled signaling is a promising target to improve infarct healing and limit post-MI remodeling.

Laboratory Investigation (2016) 96, 168-176; doi:10.1038/labinvest.2015.139; published online 14 December 2015

Although left ventricular (LV) remodeling after myocardial infarction (MI) is aimed at maintaining cardiac pump function, initial infarct size, and the subsequent progressive expansion and thinning of the infarcted area constitute the main risk factors for the development of post-MI heart failure. ${ }^{1}$ Several strategies that influence either the infarct size and/or the ensuing process of LV remodeling have been proposed as potential therapies to halt the development and progression of LV dysfunction. ${ }^{2,3}$

Cardiac fibroblasts, which account for up to $70 \%$ of the cells present in the myocardium, regulate extracellular matrix (ECM) turnover, and have an essential role in cardiac homeostasis. Fibroblasts are more resistant to ischemia than cardiomyocytes, ${ }^{4-6}$ and prolonged myocardial ischemia results in death of particularly the cardiomyocytes, whereas the fibroblasts survive. Fibroblasts have therefore been proposed to be a therapeutic target to influence the healing process of the infarcted myocardium. ${ }^{1,5,7-9}$ In addition to these resident fibroblasts, fibroblasts enter the infarcted tissue by migration. When present in the infarcted area, the fibroblasts gradually differentiate into their more contractile and synthetic myofibroblast phenotype. These (myo)fibroblasts have key roles both in the initial inflammatory phase as well as in the subsequent proliferative phase of the postinfarction response, which together lead to the formation of a stable, collagen-rich scar. ${ }^{4}$ Thus, increasing myofibroblast presence shortly after MI has been proposed to promote scar contraction and limit LV dilation. ${ }^{1,4,10}$ Fibroblast migration is inhibited by Wnt/Frizzled (Fzd) signaling. Targeting the Wnt signaling pathway by modulating its ligand, the Fzd receptors,

\footnotetext{
'Department of Cardiology, Division of Experimental Cardiology, Thoraxcenter Erasmus MC, University Medical Center Rotterdam, Rotterdam, The Netherlands; ${ }^{2}$ Department of Pharmacology and Toxicology, Maastricht University, Maastricht, The Netherlands and ${ }^{3}$ Department of Neonatology, Erasmus MC, Rotterdam, The Netherlands

Correspondence: Dr D Merkus, PhD, Division of Experimental Cardiology, Department of Cardiology, Thoraxcenter Erasmus MC, University Medical Center Rotterdam, PO Box 2040, Rotterdam 3000 CA, The Netherlands.

E-mail: d.merkus@erasmusmc.nl

${ }^{4}$ These authors contributed equally to this work.
}

Received 17 April 2015; revised 27 July 2015; accepted 26 August 2015 
through UM206, an antagonist of Fzd1 and Fzd2, has been shown to promote fibroblast migration, limit infarct size, and attenuate adverse remodeling after infarction induced by permanent ligation of the coronary artery in mice. ${ }^{11,12} \mathrm{~A}$ potential pitfall of altering fibroblast presence is that in the remote non-infarcted myocardium, local fibroblasts may remain activated in response to volume and pressure overload and promote interstitial fibrosis. ${ }^{1,13}$

In light of these considerations, the present study was designed to investigate whether UM206 is capable of limiting adverse LV remodeling after MI in a preclinical setting, that is, when tested in a large translational swine model of reperfused MI. Moreover, we aimed to elucidate the molecular mechanism of a potential beneficial effect of UM206 on remodeling of the infarct area as well as the remote myocardium. For this purpose, swine were subjected to $2 \mathrm{~h}$ of coronary artery occlusion followed by reperfusion. After one day, cardiac function was assessed with echocardiography and UM206 or Saline was continuously infused intra-arterially throughout the 5-week follow-up period. At follow-up, cardiac function was reassessed and myocardial tissue was analyzed to elucidate underlying mechanisms and signaling pathways.

\section{MATERIALS AND METHODS}

Animal experiments were performed in accordance with the Guide for the Care and Use of Laboratory Animals (NIH Publication No. 85-23, revised 1996) and with approval of the Erasmus Medical Center Animal Care Committee. Twenty-two preadolescent (2-3 months old) Yorkshire $\times$ Landrace swine $(21 \pm 1 \mathrm{~kg})$ of either sex were used.

\section{Surgery}

Swine were sedated with ketamine $(20 \mathrm{mg} / \mathrm{kg}$, intramuscularly) and midazolam $(0.5 \mathrm{mg} / \mathrm{kg}$, intramuscularly), anesthetized with thiopental $(10 \mathrm{mg} / \mathrm{kg}$, intravenously), intubated and ventilated with $\mathrm{O}_{2} / \mathrm{N}_{2}(1 / 3(\mathrm{v} / \mathrm{v}))$, and anesthetized with fentanyl $(20 \mu \mathrm{g} / \mathrm{kg} / \mathrm{h}){ }^{14,15}$ Following a thoracotomy through the fourth left intercostal space, a polyvinyl catheter was inserted into the aorta. The heart was exposed via a small pericardial incision, the proximal left circumflex artery was dissected, and a suture was placed around it. Subsequently, a polyvinyl catheter was inserted into the left atrium. Baseline blood samples were taken. Following administration of heparin (5000 IU intravenously), the proximal left circumflex artery was occluded for $2 \mathrm{~h}$ by ligation of the suture followed by reperfusion. Administration of heparin was repeated after $1 \mathrm{~h}$ of occlusion. Then following reperfusion, catheters were tunneled to the back, filled with heparin solution to prevent clotting, and the pericardium and the chest were closed. Blood samples were taken after $50 \mathrm{~min}$ of reperfusion. One animal died during reperfusion because of recurrent fibrillation. Eight weight-matched swine that underwent a thoracotomy with placement of a catheter in the aorta, but without ischemia-reperfusion, served as sham-operated animals. All animals were allowed to recover, receiving analgesia $(0.3 \mathrm{mg}$ buprenorphine intramuscularly) for 2 days and antibiotic prophylaxis $(25 \mathrm{mg} / \mathrm{kg}$ amoxicillin and $5 \mathrm{mg} / \mathrm{kg}$ gentamicin intravenously) for 5 days.

\section{Echocardiography, Hemodynamic Measurements, and Follow-Up}

One day after surgery, (awake) animals underwent echocardiography for the determination of LV end-diastolic cross-sectional area (EDA) and end-systolic cross-sectional area (ESA). ${ }^{16,17}$ 2D ejection fraction was calculated as $(\mathrm{EDA}-\mathrm{ESA}) / \mathrm{EDA} \times 100 \%$. Also, blood samples were taken from the aorta and collected in EDTA anticoagulation tubes, centrifuged, and plasma was stored at $-80^{\circ} \mathrm{C}$ until further analysis. Blood pressure was recorded immediately following echographic measurements using the CODAS software and analyzed using a custom written program in Matlab.

Subsequently, animals were randomly assigned to the UM206 group (seven swine) or the Control-MI group (six swine). UM206 (target dose $0.6 \mu \mathrm{g} / \mathrm{kg}$ per day via the left atrium) was continuously infused at a rate of $1 \mathrm{ml} / \mathrm{h}$, using a balloon pump (Easypump, BBraun, filled with $240 \mathrm{ml}$ saline, heparin $(100 \mathrm{IU} / \mathrm{ml}), 0.001 \mathrm{~g}$ EDTA, and $240 \mu \mathrm{g}$ UM206). In the Control-MI group, swine received an identical balloon pump but without UM206. Echographic and hemodynamic measurements, as well as blood sampling, were repeated after 3 and 5 weeks, and the balloon pump was refilled when necessary over the course of 5 weeks.

\section{Extensive hemodynamic measurements under anesthesia and tissue harvesting}

Five weeks after initial surgery, swine were reanesthetized with pentobarbital $(10-15 \mathrm{mg} / \mathrm{kg} / \mathrm{h}$, intravenously) and intubated and ventilated with a mixture of oxygen and nitrogen. A Swan-Ganz catheter was inserted via the femoral vein and advanced under fluoroscopic guidance into the pulmonary artery for the measurement of pulmonary artery pressure and cardiac output (thermodilution). A Millar catheter was inserted into the left carotid artery and advanced into the left ventricle for the measurement of LV pressure and its first derivative $(\mathrm{d} P / \mathrm{d} t)$. After completion of all measurements, animals underwent a sternotomy, the heart was arrested by electrical fibrillation, and immediately excised. The left ventricle was sectioned into transversal slices of $\sim 1-2 \mathrm{~cm}$, which were first weighed and photographed. Subsequently, tissues from the anterior wall and infarct tissue were taken and further subdivided for storage in liquid nitrogen, isopentane, and buffered formaldehyde (3.5-4\%) for further histological and molecular assessment.

\section{Infarct Mass Measurements}

Infarct mass at baseline

To assess infarct mass at baseline, heart-specific fatty acid-binding protein (hFABP) was determined with ELISA from the plasma samples obtained at $50 \mathrm{~min}$ of reperfusion according to the manufacturer's description (Life Diagnostics, 
Table 1 Infarct mass at baseline and follow-up

\begin{tabular}{lccc}
\hline & Sham & Control-MI & UM206 \\
\hline hFABP $(\mathrm{ng} / \mathrm{ml})$ & - & $141 \pm 34$ & $155 \pm 20$ \\
IM $_{\text {baseline }}(\mathrm{g})$ & - & $14.5 \pm 1.5$ & $17.7 \pm 1.9$ \\
IM & - & $14.2 \pm 2.3$ & $11.2 \pm 2.2^{\dagger}$ \\
Infarct size & - & $10.6 \pm 1.7$ & $8.7 \pm 1.6$ \\
LVM/BM (g/kg) & $2.31 \pm 0.10$ & $3.35 \pm 0.21^{*}$ & $3.14 \pm 0.39^{*}$ \\
RVM/BM (g/kg) & $0.75 \pm 0.04$ & $1.11 \pm 0.06^{*}$ & $1.13 \pm 0.09^{*}$ \\
& & & \\
\hline
\end{tabular}

BM, body mass; FU, follow-up; hFABP, heart-specific fatty acid-binding protein; IM, infarct mass; LVM, left ventricular mass; MI, myocardial infarction; RVM, right ventricular mass.

Data are expressed as mean \pm s.e.m. ${ }^{*} P<0.05$ vs sham; ${ }^{\dagger} P<0.05$ vs corresponding baseline.

West Chester, PA, USA). Baseline infarct mass ( $\mathrm{IM}_{\text {baseline }}$ ) was estimated using the relationship between hFABP and infarct size obtained in a previous study from our laboratory. ${ }^{18}$

\section{Infarct mass at follow-up}

Infarct mass at follow-up was determined using the pictures of the individual rings of the heart by an experienced technician (blinded to treatment of the animals) using planimetry. Infarct area on basal and apical side of each ring, expressed as the percentage of total area of the ring, was averaged and multiplied by weight of the ring. Total infarct mass at follow-up $\left(\mathrm{IM}_{\mathrm{FU}}\right)$ was calculated as the sum of the weights of the infarcts in the individual rings.

\section{ELISA, Histology, and Molecular Studies}

Longitudinal circulating markers for collagen turnover

Blood samples taken at preinfarction baseline, $24 \mathrm{~h}$ postinfarction, and every week until 5-week follow-up were quantified for swine MMP-9 and TIMP-1 using ELISA according to the manufacturer's instructions (Cusabio, Huissen, The Netherlands).

\section{Histology}

To quantify myofibroblast numbers in the infarct area, paraffin-embedded sections of $4 \mu \mathrm{m}$ were stained for $\alpha$-smooth muscle actin ( $\alpha$-SMA) as described before ${ }^{19} \quad(\alpha$-SMA monoclonal antibody; Sigma, Zwijndrecht, The Netherlands). Next, the infarcted area was planimetrically quantified for myofibroblast area in a blinded matter with vessels excluded (Qwin, Leica, Rijswijk, The Netherlands). Data were expressed as myofibroblast area/total infarct area (\%).

\section{$R T-q P C R$}

Cryopreserved infarct tissue was homogenized and RNA was isolated using the RNeasy Fibrous Tissue Mini Kit (Qiagen, Hilden, Germany) according to the manufacturer's instructions. Isolated RNA was assessed for concentration and purity $\left(A_{260} / A_{280}\right.$ ratio $)$ with a NanoDrop spectrophotometer
(Thermo Fischer Scientific, USA). Next, RNA was reverse transcribed into cDNA, using the iScript cDNA Synthesis Kit (Bio-Rad, USA). Temperature gradient optimization studies were performed with pooled samples from non-infarct tissue cDNA $(n=12)$. IQ SYBR Green Supermix (Bio-Rad) was used for the detection of cDNA levels. Quantification of gene expression of genes related to either myofibroblast presence, regulation, or differentiation were quantified, as well as genes involved in ECM turnover and Wnt/Fzd signaling was performed using the comparative $\mathrm{Ct}(\Delta \mathrm{Ct})$ method, and results are expressed as ratios to the housekeeping gene cyclophilin and normalized to the average of sham-operated animals. See Supplementary Table S1 for primer details.

\section{Statistical Analysis}

Statistical analysis was performed in SPSS. Expression data were first compared between sham, Control-MI remote, and UM206-treated remote myocardium using ANOVA. Subsequently, as infarct mass variation at baseline was considerable, ranging from 9.6 to $23.5 \mathrm{~g}$, ANCOVA with UM206 as a factor and infarct size determined by plasma hFABP as a covariate was used. When hFABP was not related to expression, an ANOVA for repeated measures was used with area (infarct $v s$ remote) as within-subject factor and UM206 treatment as between-subject factor. Hemodynamic and echographic data were analyzed using ANOVA or ANCOVA (with infarct size as covariate) for repeated measures with time as within-subject factor and group as between-subject factor. Data are given as mean \pm s.e.m. $P<0.05$ was considered statistically significant.

\section{RESULTS}

Occlusion of the proximal left circumflex artery resulted in infarction of the lateral wall of the left ventricle as evidenced by marked changes in the ECG (not shown) and hFABP release. Although plasma hFABP concentrations at 50 min of reperfusion varied considerably between the individual animals, overall plasma hFABP concentrations were similar in the Control-MI group and the UM206 group (Table 1). Estimated $\mathrm{IM}_{\text {baseline }}$ was therefore also similar between groups.

Treatment with UM206 for 5 weeks resulted in a significant decrease in IM, whereas IM remained unchanged in the Control-MI group (Figures 1a-c and Table 1). The alterations in infarct remodeling were accompanied by changes in LV geometry as measured with echo in awake swine. After 3 weeks follow-up, LV dilation occurred as evidenced by the larger increase in LV-EDA in both Control-MI and UM206treated swine as compared with sham-operated animals (Figures 1d-f and Table 2). This increase in LV-EDA was progressive between 3 and 5 weeks after MI in the Control-MI group, whereas LV-EDA remained constant in the UM206 group (Table 2), resulting in a significant reduction in dilation of the LV by UM206 treatment 5 weeks after MI (Table 2 and Figure 1f). This reduction in LV dilation induced by UM206 

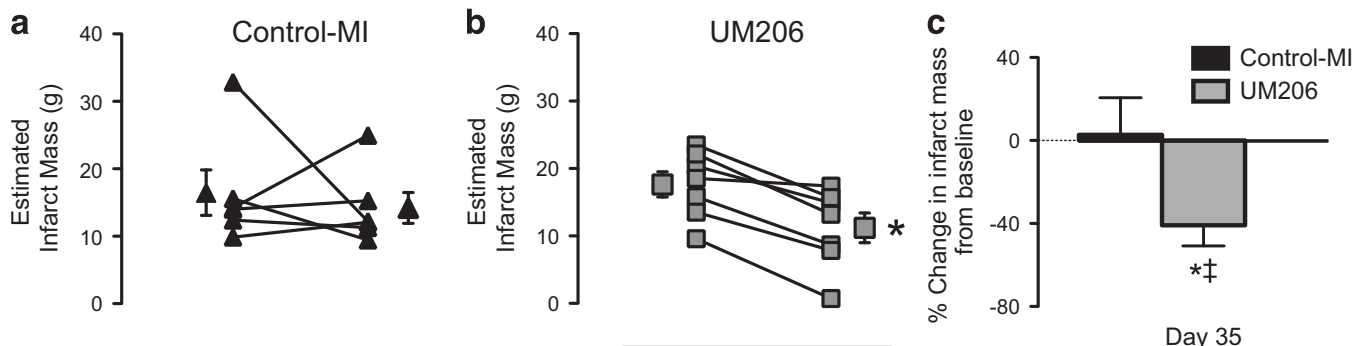

Baseline Follow-up

Baseline Follow-up
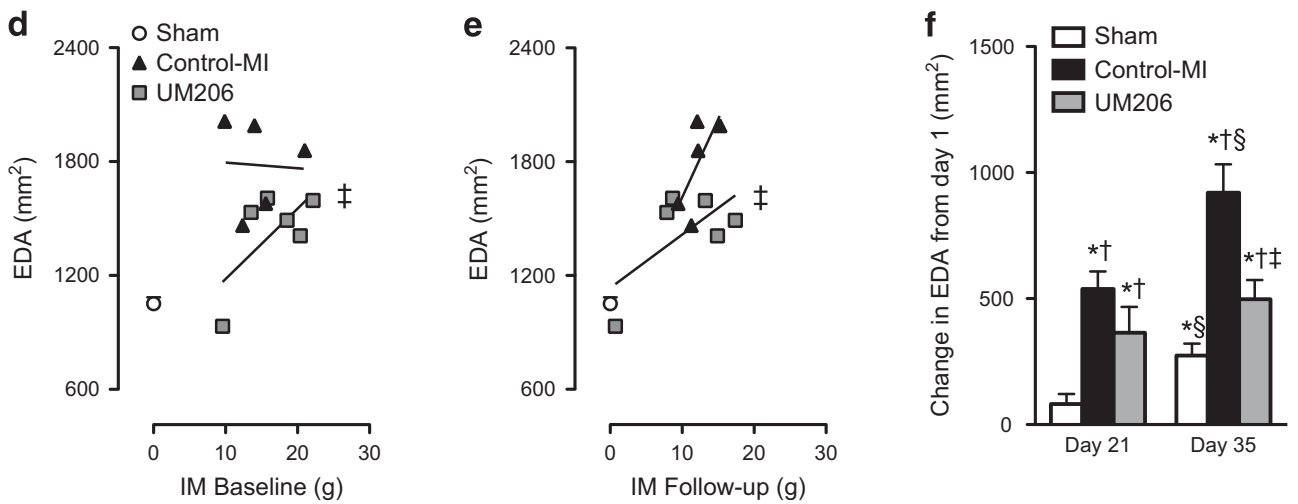

Figure 1 Effect of UM206 treatment on infarct size and left ventricular dilatation. Infarct mass as estimated by heart-specific fatty acid-binding protein (hFABP) at baseline and with planimetry at follow-up was not different in Control-myocardial infarction (MI) swine (a) but decreased significantly in UM206-treated swine (b). The relative change in infarct mass was significantly higher in UM-206-treated swine as compared with Control-MI (c). The reduction in infarct mass was accompanied by a reduced left ventricular end-diastolic area (EDA) as measured with echocardiography both when related to infarct mass (IM) at baseline (d) and at follow-up (e). This reduced dilation occurred particularly between 3 and 5 weeks after MI, when EDA increased further in Control-MI, but not in UM206-treated swine. ${ }^{*} P<0.05$, vs baseline (b and $\mathbf{c}$ ) or day 1 post-MI (f), ${ }^{\dagger} P<0.05$ vs sham, ${ }^{\ddagger} P<0.05$ UM206 treatment vs Control-MI, ${ }^{\S} P<0.05$ day 35 vs day 21.

was further supported by a rotation of the relation between LV-EDA and both $\mathrm{IM}_{\mathrm{BL}}$ (Figure 1d) and $\mathrm{IM}_{\mathrm{FU}} 5$ weeks after MI (Figure 1e). MI resulted in a significant decrease in ejection fraction at 3 and 5 weeks follow-up, but there was no overall difference in ejection fraction between UM206-treated and Control-MI swine (Table 2), due to the large variation in IM. However, $\mathrm{IM}_{\mathrm{FU}}$ was linearly related to $\mathrm{LV}$ ejection fraction (not shown), which may suggest that the IM reduction by UM206 could result in improved LV function. Hemodynamics, as measured under anesthesia, were similar between groups (Table 3), although heart rate was slightly lower in vehicle-treated swine. This was most likely the result of anesthesia, as awake heart rates were not different between groups (not shown).

In agreement with previous observations in mice, MI resulted in the occurrence of myofibroblasts in the infarct area, activation of the Wnt/Fzd pathway, inflammation, and increased expression of ECM proteins in the infarct area. Interestingly, this occurred not only in the infarcted myocardium but also in the remote myocardium. mRNA for Wnt3a was reduced in the remote, non-infarcted myocardium as compared with sham-operated swine, and was increased in the infarcted area as compared with the remote area (Table 4). mRNA for both Fzd2 and Fzd4 was increased in remote non-infarcted myocardium as compared with sham, and while expression of Fzd2 tended to be more increased $(P=0.06)$ in the infarcted area, Fzd4 mRNA expression was less in infarcted as compared with the remote area (Table 4). mRNA expression of the Fzd co-receptor LRP5 was increased, whereas mRNA expression of the co-receptor LRP6 was decreased in remote as well as in infarcted myocardium as compared with sham-operated animals. TGF- $\beta 1$ and collagen-3 were increased in remote and infarcted myocardium of swine with MI, whereas collagen-1 was slightly lower in remote myocardium of swine with MI as compared with sham-operated animals, but was increased in the infarcted myocardium in comparison with remote myocardium (Table 4). Moreover, LOX, one of the enzymes responsible for collagen-crosslinking, as well as tenascin-C, a glycoprotein that is expressed in the ECM following injury, were increased in the infarcted myocardium as compared with remote tissue. In the downstream part of the Wnt/Fzd signaling pathway, axin and APC were unaltered in either remote or infarcted myocardium, whereas $\beta$-catenin was increased in remote and infarcted myocardium of both Control-MI swine and swine treated with UM206 as compared with sham-operated swine.

The infarct reduction by UM206 was accompanied by changes in infarct composition and expression of genes involved in ECM remodeling and Wnt/Fzd signaling. 
Table 2 Echocardiographic parameters

\begin{tabular}{lccc}
\hline Days post-MI & Sham & Control-MI & UM206 \\
\hline Day 1 & & & \\
EDA $\left(\mathrm{mm}^{2}\right)$ & $778 \pm 49$ & $851 \pm 75$ & $902 \pm 84$ \\
ESA $\left(\mathrm{mm}^{2}\right)$ & $359 \pm 22$ & $448 \pm 86$ & $456 \pm 60$ \\
EF (\%) & $53 \pm 2$ & $49 \pm 6$ & $50 \pm 3$ \\
& & & \\
Day 21 & & & $1266 \pm 81^{*}$ \\
EDA (mm $\left.{ }^{2}\right)$ & $860 \pm 50$ & $843 \pm 67^{*}$ & $693 \pm 61^{*}$ \\
ESA (mm $\left.{ }^{2}\right)$ & $360 \pm 41$ & $39 \pm 4^{*}$ & $45 \pm 4^{*}$ \\
EF (\%) & $58 \pm 4$ & & \\
& & $1781 \pm 110^{*}$ & $1428 \pm 103^{*,+}$ \\
Day 35 & & $1010 \pm 95^{*}$ & $789 \pm 113^{*}$ \\
EDA (mm $\left.{ }^{2}\right)$ & $1052 \pm 33$ & $44 \pm 3^{*}$ & $45 \pm 5$ \\
ESA (mm $\left.{ }^{2}\right)$ & $471 \pm 53$ & & \\
EF (\%) & $56 \pm 4$ & & \\
\hline
\end{tabular}

$E D A$, end diastolic area; $E F$, ejection fraction; $E S A$, end systolic area; $\mathrm{MI}$, myocardial infarction.

Data are expressed as mean \pm s.e.m. ${ }^{*} P<0.05$ vs sham; ${ }^{\dagger} P<0.05$ vs Control-MI.

Histological assessment of $\alpha$-SMA-positive cells in the infarcted myocardium revealed a significantly lower presence of myofibroblasts in the UM206 group (Figure 2a), which when also corrected for infarct mass at baseline was corroborated by a trend towards a reduction in $\alpha$-SMA mRNA expression $(P=0.076)$. Interestingly, the amount of myofibroblasts present correlated very well with the change in IM over time in the Control-MI group, but not in the UM206-treated group (Figure 2b). In accordance with their role in ECM turnover, the reduced presence of myofibroblasts resulted in a decrease in TIMP-1 (Figure 2c) and a trend towards a decrease in MMP-9 mRNA $(P=0.085$; Figure 2d) in the UM206-treated group as compared with the Control-MI group 5 weeks after induction of MI. The effects of UM206 treatment on local TIMP-1 and MMP-9 expression were not reflected in significant changes in MMP-9 and TIMP-1 in circulating plasma. TIMP-1 levels were below the detection limit of the assay in most plasma samples and were not different between Control-MI and UM206-treated swine. MI resulted in a significant increase in plasma MMP-9 one day post-MI before the start of UM206 treatment $(15 \pm 5 \mathrm{ng} / \mathrm{ml}$ at day 1 vs $8 \pm 4 \mathrm{ng} / \mathrm{ml}$ just before initiation of MI) but was not different between groups. This increase in plasma MMP-9 tended to wane over time, being $6 \pm 1 \mathrm{ng} / \mathrm{ml}$ at 5 weeks follow-up ( $P=0.10$ vs day 1$)$, but serial assessment of plasma MMP-9 did not show any differences between Control-MI and UM206-treated swine.

Interestingly, mRNA expression of Fzd4 was significantly higher in the infarcted myocardium of the UM206-treated
Table 3 Hemodynamic parameters under anesthesia

\begin{tabular}{lccc}
\hline & Sham & Control-MI & UM206 \\
\hline Heart rate (b.p.m.) & $99 \pm 3$ & $83 \pm 7^{*}$ & $103 \pm 7$ \\
Aorta pressure $(\mathrm{mm} \mathrm{Hg})$ & $92 \pm 5$ & $103 \pm 7$ & $103 \pm 2$ \\
$\mathrm{LVSP}(\mathrm{mm} \mathrm{Hg})$ & $103 \pm 4$ & $118 \pm 8$ & $115 \pm 2$ \\
$\mathrm{LVEDP}(\mathrm{mm} \mathrm{Hg})$ & $13 \pm 2$ & $15 \pm 3$ & $13 \pm 2$ \\
$\mathrm{dP} / \mathrm{d} t_{\max }(\mathrm{mm} \mathrm{Hg} / \mathrm{s})$ & $1620 \pm 140$ & $1690 \pm 180$ & $1590 \pm 160$ \\
$\mathrm{dP} / \mathrm{d} t_{\min }(\mathrm{mm} \mathrm{Hg} / \mathrm{s})$ & $-1600 \pm 140$ & $-2100 \pm 140$ & $-2100 \pm 170$ \\
$\mathrm{CO}(\mathrm{I} / \mathrm{min})$ & $3.9 \pm 0.3$ & $3.4 \pm 0.5$ & $4.2 \pm 0.3$ \\
\hline
\end{tabular}

$\mathrm{CO}$, cardiac output; $\mathrm{dP} / \mathrm{d} t_{\text {max, }}$ maximal rate of rise of left ventricular pressure; $\mathrm{dP} / \mathrm{d} t_{\min }$, maximal rate of decrease in left ventricular pressure; LVEDP, left ventricular end diastolic pressure; LVSP, left ventricular systolic pressure; Ml, myocardial infarction.

Data are expressed as mean \pm s.e.m. ${ }^{*} P<0.05$ vs sham; ${ }^{\dagger} P<0.05$ vs Control-MI.

animals (Table 4). This higher expression of Fzd4, a receptor thought to be involved in angiogenesis, was accompanied by a tendency towards a higher expression of VEGF in the infarcted myocardium of UM206-treated animals as compared with Control-MI, whereas expression of the VEGF receptors Flt1 and KDR was reduced in infarcted area as compared with remote myocardium, but not further affected by UM206 treatment.

mRNA for the Fzd co-receptors LRP5 $(P=0.06)$ and LRP6 $(P<0.05)$ was higher in the remote myocardium of UM206treated animals as compared with Control-MI (Table 4), which was accompanied by a trend towards a lower TIMP-1 expression $(P=0.07)$, suggesting that UM206 treatment may directly affect remodeling of the remote myocardium as well.

\section{DISCUSSION}

In the present study, the effect of UM206, a modulator of Wnt/Fzd signaling, was investigated on infarct remodeling and LV function in a clinically relevant large animal model. The most important findings in the present study are that treatment with UM206 for 5 weeks after acute MI, resulted in (i) IM reductions and (ii) reduced dilation of the LV, which was accompanied by (iii) changes in the expression of genes involved in ECM remodeling and Wnt/Fzd signaling. The implications of these findings will be discussed below.

\section{Myofibroblasts and LV Remodeling After MI}

MI results in dilation and remodeling of the left ventricle. Although this process is aimed at maintaining pump function, dilation of the left ventricle has been shown to be an initiating factor in the process leading to heart failure. Infarct size and composition of the scar tissue, replacing the infarcted myocardium, are important determinants of the outcome of the LV remodeling process. To assess accurately the effect of treatment, in a study with relatively few animals, it is important to not only measure infarct size at the end of the 
Table 4 RT-qPCR for Wnt signaling, structural extracellular matrix components, and growth factors

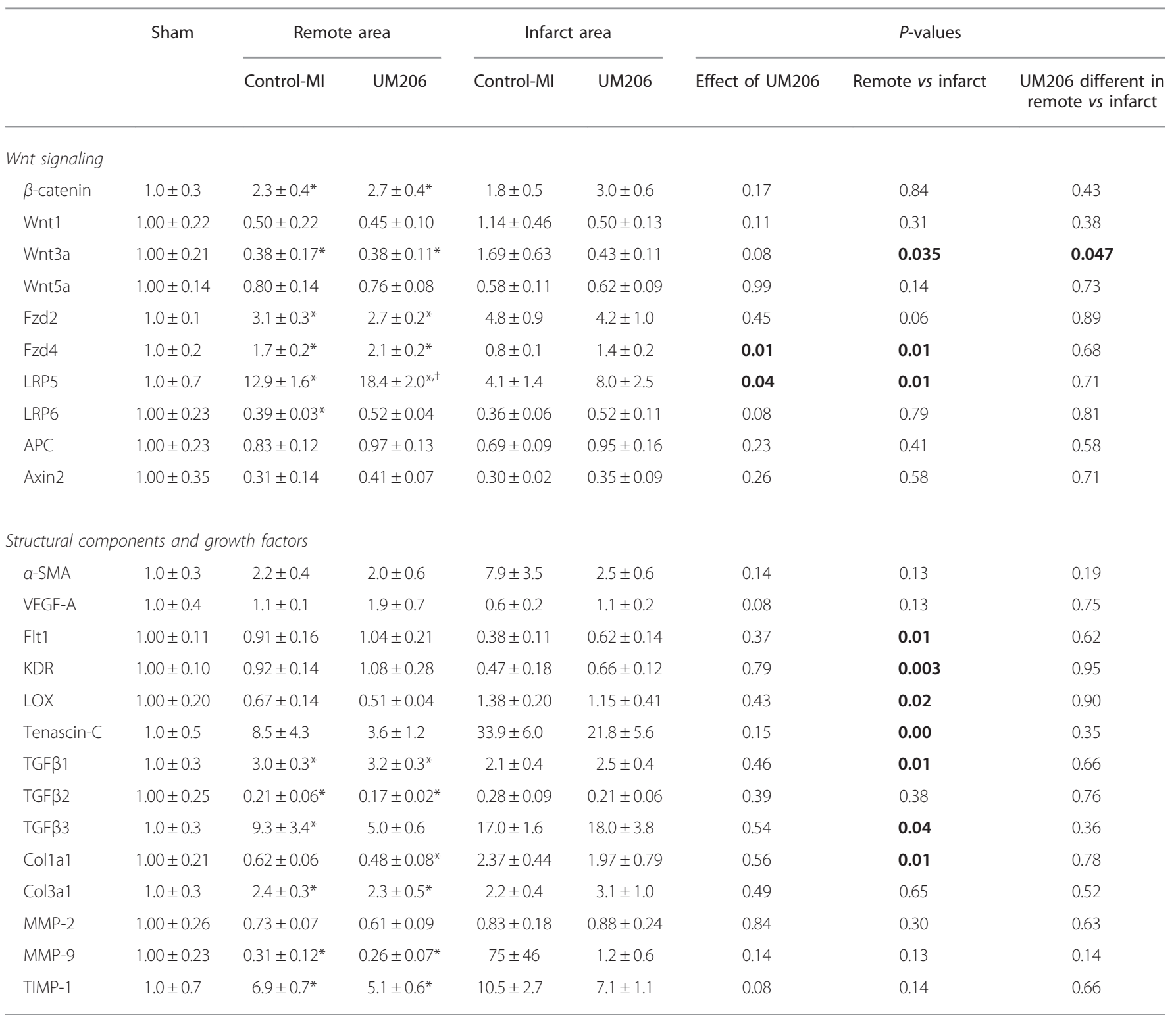

ANOVA, analysis of variance; APC, adenomatous polypolis coli; Axin, axis inhibition protein; $a$-SMA, $a$-smooth muscle actin; Col, collagen; Flt1, Fms-related tyrosine kinase-1; Fzd, frizzled; KDR, kinase insert domain receptor; LOX, lysil oxidase; LRP, low-density lipoprotein receptor-related protein; $\mathrm{Ml}$, myocardial infarction; MMP, matrix metalloproteinase; RM, repeated measures; RT-qPCR, reverse transcription-quantitative polymerase chain reaction; TGF, transforming growth factor; TIMP, tissue inhibitor of metalloproteinases; VEGF-A, vascular endothelial growth factor A.

Data are ratios to housekeeping gene cyclophilin and are displayed relative to sham values \pm s.e.m.; sham: $n=8$; Control-MI: $n=6$; UM206: $n=6$. ${ }^{*} P<0.05$ vs sham, ${ }^{\dagger} P<0.05$ vs Control-MI by ANOVA between sham and remote area of Control-MI and UM206. $P$-values in last three columns are from RM-ANOVA between remote and infarct area of Control-MI and UM206-treated animals. Significant $P$-values are denoted in bold.

study but also at baseline, and to correlate findings at the end of the study to the factor that initiated the remodeling process. In a recent study, we found that hFABP, when measured in blood at $50 \mathrm{~min}$ of reperfusion, correlated linearly with infarct mass as determined by TTC staining following killing of the animals. ${ }^{18}$ In the present study, hFABP correlated well with the clinical marker high-sensitive troponin I $(r=0.72)$, further confirming the validity of our estimation of infarct mass at baseline. $\mathrm{IM}_{\text {baseline, estimated by }}$ measuring hFABP, did vary between individual animals, but overall was similar between Control-MI and UM206-treated groups.

Infarct healing occurs in three partly overlapping phases: an initial inflammatory phase, followed by a proliferative phase and maturation of the scar. (Myo)fibroblasts have different roles in the different phases. ${ }^{4,10}$ In the initial phase, the fibroblasts act as local immune modulators and are the main effectors of fibrogenesis. ${ }^{1,4-6,20}$ In the subsequent proliferative 

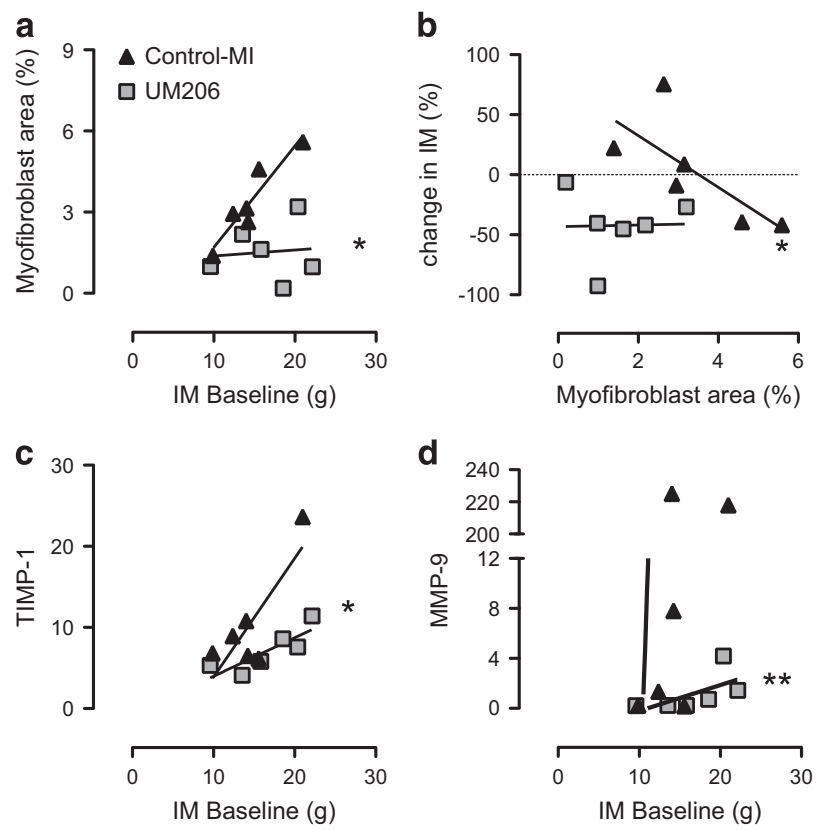

Figure 2 Effect of UM206 treatment on infarct remodeling and expression of markers for myofibroblast presence and extracellular matrix remodeling. UM206 reduced myofibroblast presence as assessed with immunohistochemistry in the infarcted area $(\mathbf{a}, 1.53 \pm 0.43 \%$ in UM206 vs $3.38 \pm 0.61 \%$ in Control-myocardial infarction (MI)). Myofibroblast presence correlated with the change in infarct mass (IM) over time, with higher myofibroblast presence being associated with a larger reduction in IM in Control-MI swine (b). The lower myofibroblast presence in UM206-treated swine suggests that myofibroblast-induced IM reduction was already completed in this group. The reduced presence of myofibroblasts was associated with a decrease in tissue inhibitor of metalloproteinase-1 (TIMP-1) (c) and a trend towards a reduction in matrix metalloproteinase9 (MMP-9) (d). ${ }^{*} P<0.05,{ }^{*} P<0.10$ UM206 treatment vs Control-MI.

phase of healing, fibroblasts further differentiate into myofibroblasts that not only have an augmented matrixsynthetic phenotype but also express the contractile protein $\alpha$-SMA. Both the increased ECM production and the contractile properties of the myofibroblasts have been proposed to be key factors in strengthening the infarct and limiting infarct expansion, thereby reducing LV dilation, ${ }^{1,4,9,21}$ and therefore constitute a target to modulate infarct remodeling. Administration of UM206 promotes migration of fibroblasts to the infarcted area by inhibition of Wnt/Fzd signaling, and thereby modulates the scar formation in the infarcted area. ${ }^{11}$ In accordance with our recent study in mice, ${ }^{11}$ we found in the present study that treatment with UM206 for 5 weeks reduced infarct mass as compared with its baseline measurement. This reduction in infarct mass was accompanied by a decreased LV dilation, as measured in awake animals with echocardiography, between 3 and 5 weeks after MI in swine treated with UM206. Interestingly, $\mathrm{IM}_{\mathrm{FU}}$ correlated very well with ejection fraction measured at 5 weeks follow-up, which may suggest that, although there was no overall difference in ejection fraction between Control-MI and UM206-treated swine because of variation in infarct size, by promoting infarct reduction, UM206 treatment may improve cardiac function.

In both the canine ${ }^{22}$ and the porcine ${ }^{10}$ heart, myofibroblast presence peaks between 5 and 21 days after MI, after which myofibroblast numbers gradually decline. This has led to the concept that during the phase of infarct maturation, when the infarct is filled with matrix, myofibroblast proliferation is suppressed, and myofibroblasts become quiescent and undergo apoptosis. ${ }^{4}$ In the present study, we found that the presence of myofibroblasts, as indicated by immunohistochemistry (in which we excluded blood vessels) as well as mRNA expression (that may in part reflect $\alpha$-SMA from blood vessels) for $\alpha$-SMA, was higher in the infarcted tissue as compared with remote tissue and correlated not only with $\mathrm{IM}_{\mathrm{BL}}$ but also with the estimated change in IM over time in the Control-MI group, suggesting that, indeed, myofibroblasts contribute to infarct contraction. In contrast to our previous study in mice, in which UM206 treatment increased myofibroblast presence in the infarcted area, ${ }^{11}$ myofibroblast presence was lower in the infarcted myocardium of UM206treated swine as compared with Control-MI, particularly in swine with a large MI, despite the observation that the reduction in IM was larger. It should be noted, however, that infarct size as a percentage of the left ventricle in mice is much larger than the infarct size in swine in the present study, and therefore mechanical stress within the murine infarcts is expected to be larger. As myofibroblast differentiation is promoted by mechanical stress, ${ }^{23}$ it is likely that the UM206-induced infarct remodeling earlier after MI attenuated mechanical stress 5 weeks after MI, and thereby reduced myofibroblast presence. This is in accordance with a recent study in swine showing that, in control infarcts myofibroblasts presence increased between 7 and 21 days of infarct, whereas treatment with a biocomposite material resulted in an increased myofibroblast presence 7 days after MI, while myofibroblasts were lower at 21 days, at a time when LV dilation was reduced compared with control infarcts. ${ }^{10}$ Importantly, it has been suggested that persistent myofibroblast presence in the injured heart, particularly in the remote non-infarcted myocardium, may be detrimental as it may lead to excessive fibrosis and contribute to heart failure. ${ }^{1,24}$ It is therefore important to also evaluate changes in the remote myocardium. MMP-9 was decreased while TIMP-1 and collagen-3 were increased in the remote myocardium after MI, which is suggestive of increased interstitial fibrosis, ${ }^{1,13}$ but UM206 treatment did not modulate this expression.

In humans, LV dilation was shown to correlate with circulating MMP-9 levels. $^{25,26}$ In our study, however, biological activity of early myofibroblast presence was not reflected in circulating levels of MMP-9 and TIMP-1, typical markers for collagen turnover. It is possible that the surgical trauma in combination with the chronic instrumentation of our animals evoked inflammatory and reparative processes that influenced the plasma levels of MMP-9, which may have masked the effect of the MI. Moreover, plasma levels of 
MMP-9 do not only reflect the increased expression of MMP-9 in the infarcted area but are also influenced by the decreased expression of MMP-9 in the remote-non-infarcted myocardium. Thus, it is likely that local measures for wound healing more accurately assess the wound-healing process as compared with circulating ones. Indeed, in contrast to the unaltered circulating plasma levels of MMP-9, MMP-9 mRNA tended to be higher in the infarcted myocardium of Control-MI as compared with UM206-treated swine 5 weeks after MI and correlated with infarct size, which is in accordance with the increased presence of myofibroblasts, and their role in modulation of the ECM. In combination with our observation that expression of collagens 1 and 3 was unaltered by UM206, the increased levels of MMP-9 in the Control-MI swine may have resulted in enhanced degradation of the ECM, which could have contributed to the LV dilation that occurred between 3 and 5 weeks after MI.

Almost all Fzd members have been identified in the healthy heart tissue but signaling in the adults is silent. ${ }^{20,24}$ The Wnt/Fzd signaling pathway is activated in wound healing after MI. ${ }^{21,27}$ In the present study, Wnt3a as well as Fzd receptor expression patterns were indeed different in remote $v s$ infarcted myocardium. Most of our findings regarding changes in activation of Wnt/Fzd signaling are in accordance with a recently published study in swine in which gene expression was evaluated using microarrays. ${ }^{28}$ Thus, Fzd2 was increased, whereas Fzd4 was decreased in infarcted myocardium as compared with remote myocardium. Wnt3a was upregulated in the infarcted myocardium in the present study but downregulated in this previous study at 3 weeks after MI, ${ }^{28}$ whereas unchanged at 6 weeks after MI. ${ }^{28}$ Also, downstream targets in the Wnt/Fzd signaling cascade were similarly affected by MI; Axin2 was decreased, whereas APC was unaltered. ${ }^{28}$ UM206 treatment reduced the upregulation of Wnt3a in the infarcted tissue. As Wnt3a promotes myofibroblast differentiation, ${ }^{29}$ this observation is in accordance with the presence of myofibroblasts that is reduced in UM206-treated as compared with Control-MI swine.

Although, in the present study, we focused on the effect of altering Wnt/Fzd signaling in myofibroblasts, it is possible that altering signaling in other cell types within the myocardium may have contributed to the beneficial effects of UM206. Wnt signaling has also been related to inflammation and angiogenesis, ${ }^{7}$ and expression of Fzd4, a receptor that has been shown to be involved in stabilization of microvessels, was higher in animals treated with UM206 as compared with Control-MI. As microvessel stabilization is protective against microvascular regression in the maturation phase of MI, the increased expression of Fzd4 may have contributed to the improved infarct healing.

\section{CONCLUSION}

Administration of the peptide fragment UM206 attenuates the dilation of the left ventricle after MI in a translationally relevant swine model of ischemia-reperfusion. From this observation, we conclude that inhibition of Wnt/Fzd signaling has a beneficial effect on the wound healing after MI, resulting in reduced adverse remodeling of the heart. Although the effects on cardiac function were limited at the 5-week time point, it is attractive to speculate that prolonged administration of UM206 will prevent the deterioration of the cardiac performance that is frequently observed after infarction.

Supplementary Information accompanies the paper on the Laboratory Investigation website (http://www.laboratoryinvestigation.org)

\section{ACKNOWLEDGMENTS}

We gratefully acknowledge technical assistance of A Verzijl, S Sneep, $M$ te Lintel Hekkert, L Vervoort-Peters and LA Blonden. This study was partially supported by a grant from the Dutch Heart Foundation (2010B196), Den Haag, The Netherlands, Cyttron II (FES0908), and CTMM-TRIUMPH.

\section{DISCLOSURE/CONFLICT OF INTEREST}

The authors declare no conflict of interest.

1. Daskalopoulos EP, Hermans KC, Blankesteijn WM. Cardiac (myo) fibroblast: novel strategies for its targeting following myocardial infarction. Curr Pharm Des 2014;20:1987-2002.

2. Kloner RA, Jennings RB. Consequences of brief ischemia: stunning, preconditioning, and their clinical implications: part 1. Circulation 2001;104:2981-2989.

3. Kloner RA, Jennings RB. Consequences of brief ischemia: stunning, preconditioning, and their clinical implications: part 2. Circulation 2001;104:3158-3167.

4. Chen W, Frangogiannis NG. Fibroblasts in post-infarction inflammation and cardiac repair. Biochim Biophys Acta 2013;1833:945-953.

5. Ma $\mathrm{Y}$, de Castro Bras LE, Toba $\mathrm{H}$ et al. Myofibroblasts and the extracellular matrix network in post-myocardial infarction cardiac remodeling. Pflugers Arch 2014;466:1113-1127.

6. Turner NA, Porter KE. Function and fate of myofibroblasts after myocardial infarction. Fibrogenesis Tissue Repair 2013;6:5.

7. Hermans KC, Daskalopoulos EP, Blankesteijn WM. Interventions in Wnt signaling as a novel therapeutic approach to improve myocardial infarct healing. Fibrogenesis Tissue Repair 2012;5:16.

8. Porter KE, Turner NA. Cardiac fibroblasts: at the heart of myocardial remodeling. Pharmacol Ther 2009;123:255-278.

9. Hermans KC, Blankesteijn WM. Wnt signaling in cardiac disease. Compr Physiol 2015;5:1183-11209.

10. McGarvey JR, Pettaway S, Shuman JA et al. Targeted injection of a biocomposite material alters macrophage and fibroblast phenotype and function following myocardial infarction: relation to left ventricular remodeling. J Pharmacol Exp Ther 2014;350:701-709.

11. Laeremans $\mathrm{H}$, Hackeng TM, van Zandvoort MA et al. Blocking of frizzled signaling with a homologous peptide fragment of wnt3a/ wnt5a reduces infarct expansion and prevents the development of heart failure after myocardial infarction. Circulation 2011;124: 1626-1635.

12. Hermans KC, Daskalopoulos E, Janssen BJ et al. UM206, a frizzled-receptor antagonist attenuates adverse remodeling and cardiac function deterioration following myocardial infarction. FASEB J 2014;28(Suppl):652.610.

13. Shinde AV, Frangogiannis NG. Fibroblasts in myocardial infarction: a role in inflammation and repair. J Mol Cell Cardiol 2014;70:74-82.

14. Boontje NM, Merkus D, Zaremba R et al. Enhanced myofilament responsiveness upon beta-adrenergic stimulation in post-infarct remodeled myocardium. J Mol Cell Cardiol 2011;50:487-499.

15. Zhou ZC, de Wijs-Meijler D, Lankhuizen I et al. Blunted coronary vasodilator response to uridine adenosine tetraphosphate in postinfarct remodeled myocardium is due to reduced $\mathrm{P} 1$ receptor activation. Pharmacol Res 2013;77:22-29. 
16. Kuster DWD, Merkus D, Kremer A et al. Left ventricular remodeling in swine after myocardial infarction: a transcriptional genomics approach. Basic Res Cardiol 2011;106:1269-1281.

17. van der Velden J, Merkus $D$, Klarenbeek $B R$ et al. Alterations in myofilament function contribute to left ventricular dysfunction in pigs early after myocardial infarction. Circ Res 2004;95:e85-e95.

18. Uitterdijk A, Sneep S, van Duin RW et al. Serial measurement of hFABP and high-sensitivity troponin I post-PCI in STEMI: how fast and accurate can myocardial infarct size and no-reflow be predicted? Am J Physiol Heart Circ Physiol 2013;305:H1104-H1110.

19. van den Borne SW, van de Schans VA, Strzelecka AE et al. Mouse strain determines the outcome of wound healing after myocardial infarction. Cardiovasc Res 2009;84:273-282.

20. Daskalopoulos EP, Janssen BJ, Blankesteijn WM. Myofibroblasts in the infarct area: concepts and challenges. Microsc Microanal 2012;18: 35-49.

21. Daskalopoulos EP, Janssen BJ, Blankesteijn WM. Targeting Wnt signaling to improve wound healing after myocardial infarction. Methods Mol Biol 2013;1037:355-380.

22. Frangogiannis NG, Michael LH, Entman ML. Myofibroblasts in reperfused myocardial infarcts express the embryonic form of smooth muscle myosin heavy chain (SMemb). Cardiovasc Res 2000;48: 89-100.
23. Hinz B, Gabbiani G. Mechanisms of force generation and transmission by myofibroblasts. Curr Opin Biotechnol 2003;14:538-546.

24. Lajiness JD, Conway SJ. Origin, development, and differentiation of cardiac fibroblasts. J Mol Cell Cardiol 2014;70:2-8.

25. Kelly D, Cockerill G, Ng LL et al. Plasma matrix metalloproteinase-9 and left ventricular remodelling after acute myocardial infarction in man: a prospective cohort study. Eur Heart J 2007;28: 711-718.

26. Squire IB, Evans J, Ng LL et al. Plasma MMP-9 and MMP-2 following acute myocardial infarction in man: correlation with echocardiographic and neurohumoral parameters of left ventricular dysfunction. J Card Fail 2004;10:328-333.

27. Daskalopoulos EP, Hermans KC, Janssen BJ et al. Targeting the Wnt/frizzled signaling pathway after myocardial infarction: a new tool in the therapeutic toolbox? Trends Cardiovasc Med 2013;23: 121-127.

28. Prat-Vidal C, Galvez-Monton C, Nonell L et al. Identification of temporal and region-specific myocardial gene expression patterns in response to infarction in swine. PLoS One 2013;8:e54785.

29. Carthy JM, Garmaroudi FS, Luo $Z$ et al. Wnt3a induces myofibroblast differentiation by upregulating TGF-beta signaling through SMAD2 in a beta-catenin-dependent manner. PLoS One 2011;6:e19809. 